\title{
Screening of Suspected Liquids to Alcoholic Drinks by a Qualitative Chemical Kit
}

Hossein Mahdavi SA', Hassanian-Moghaddam $\mathbf{H}^{2,3}$, Hashemi Nazari SS ${ }^{4}$, Akhgari M1, Shahbazi $\mathbf{F}^{5}$, Nazari Kangavari $\mathbf{H}^{6}$, Akhavan Tavakoli Ar ${ }^{7}$, Rafizadeh $\mathbf{M}^{\mathbf{8}}$ and Rafizadeh $\mathbf{A}^{\mathbf{9} *}$

${ }^{1}$ Legal Medicine Research Center of Iran, Tehran, Iran ${ }^{2}$ Social Determinant of Health Research Center, Shahid Beheshti University of Medical Sciences, Tehran, Iran ${ }^{3}$ Department of Clinical Toxicology, Loghman Hakim Hospital, Shahid Beheshti University of Medical Sciences, Tehran, Iran

${ }^{4}$ Safety Promotion and Injury Prevention Research Center, Department of Epidemiology, School of Public Health and Safety, Shahid Beheshti University of Medical Sciences, Tehran, Iran

5Department of Epidemiology \& Biostatistics, School of Public Health, Hamadan University of Medical Sciences, Hamadan, Iran

${ }^{6}$ Department of Epidemiology \& Biostatistics, School of Public Health, Iran University of Medical Sciences, Tehran, Iran

${ }^{7}$ Legal Medicine Research Center, Legal Medicine

Organization, Rasht, Iran

${ }^{8}$ pharmacy faculty, Mazandran University of Medical

Sciences, Ramsar, Iran

${ }^{9}$ Department of Nursing \& Midwifery, Islamic Azad

University, Rasht Branch, Rasht, Iran

*Corresponding author: Rafizadeh A, Department of Nursing \&Midwifery, Rasht Branch of Islamic Azad University, Poletaleshan, Lakan road, Rasht, Iran

Received: April 20, 2021; Accepted: May 20, 2021;

Published: May 27, 2021

\section{Abbreviations}

FID: Flame Ionization Detector; GC: Gas Chromatography; GC-Mass: Gas Chromatography-Mass Spectrometry; HPLC: High Performance Liquid Chromatography; LMO: Legal Medicine Organization; NPV: Negative Predictive Value; PPV: Positive Predictive Value; SPSS: Statistical package for social science; V/V: Volume/Volume

\section{Introduction}

Production, trade, and consumption, of alcoholic drinks, are prohibited in Iran and there are severe punishments for them. But, these penalties not only have been unable to decrease alcohol consumption in Iran society but also, have been lead to increase usage of poor-quality alcohol (fake and homemade) that can be accompanied by many dangers including methanol intoxication $[1,2]$. One of the tasks of the police in Iran is the confiscation of suspected liquids to alcoholic drink and the determination of their owners' legal status by measuring the ethanol concentration in them. To measure their ethanol content, the mentioned fluids are referred to Legal Medicine Organization (LMO). Based on the Iran Legal protocols, liquids with $3 \%$ volume/volume (v/v) ethanol or less are not considered being alcoholic beverages at all, and their owners should be released immediately. But, the owners of solutions contains ethanol more than $3 \% \mathrm{v} / \mathrm{v}$ must be referred to the judicial system for the continuation of the legal proceeding $[3,4]$. But, due to different reasons, measuring the amount of ethanol of samples is so timeconsuming that put makes their owners waiting for the final court order.

Like methanol, different advanced methods including HighPerformance Liquid Chromatography (HPLC), Gas ChromatographyMass Spectrometry (GC-MS), and usually Gas Chromatography (GC) are currently used to detect or quantify ethanol in different samples including alcoholic beverages [5-11]. Likewise, biological and chemical methods are used for this purpose too [12-18]. GC is usually used as a gold standard method for the quantitative analysis of alcohol in Iran and worldwide [19]. Application of these methods is almost time-consuming and costly and need very expensive devices and high technical knowledge that make them inapplicable in common laboratories with many samples per day $[1,2,5-10,19]$.

For these reasons, now a day rapid and chemical methods have
Austin J Environ Toxicol - Volume 7 Issue 2 - 2021 ISSN: 2472-372X | www.austinpublishinggroup.com Hossein Mahdavi et al. (C) All rights are reserved
Citation: Hossein Mahdavi SA, Hassanian-Moghaddam H, Hashemi Nazari SS, Akhgari M, Shahbazi F, Nazari Kangavari H6, et al. Screening of Suspected Liquids to Alcoholic Drinks by a Qualitative Chemical Kit. Austin J Environ Toxicol. 2021; 7(2): 1038. 
been widely considered for different purposes due to their simplicity, easiness, low cost, short operation time and etc [13]. Accordingly, the accessibility to an easy method for quick screening of these samples and separating negative cases from positive ones (based on a cutoff point) to avoid spending a lot of time and money can be a great advantage.

So, in this study, the ethanol content of 839 samples was measured to evaluate the efficacy of a newly designed kit. For this purpose, the GC method was used as a gold standard. Lastly, the gained results by the kit and GC methods were compared together to confirm kit ability and final conclusion.

\section{Materials and Methods}

In this study, 839 suspected liquids to alcoholic beverages were used as samples. They had been discovered by police (Gilan province, Iran) between April 2017 and May 2018 and were referred to the LMO of Rasht, Guilan province, Iran. Their ethanol contents were determined by both kit and GC methods. The obtained results by both techniques were compared together and the kit function was approved by comparison with the GC method. The technicians of each section were blind to the results obtained by another division. Statistical analysis was performed using SPSS software version 24 (IBM Corporations, Chicago, Ill, USA).

\section{Apparatus}

A GC apparatus (Shimadzum 148, Japan) was used to determine the ethanol content of the samples. This device contained a packed column and FID detector. To performance of the tests, $0.2 \mu \mathrm{L}$ of the diluted $(1: 100)$ samples were manually injected to GC by $1 \mu \mathrm{L}$ Hamilton syringe. The GC injector and detector temperatures were set at $180^{\circ} \mathrm{C}$ and $210^{\circ} \mathrm{C}$, respectively. No internal standard was applied.

\section{Chemicals}

The samples (839 suspected fluids) were prepared from alcohol lab of Rasht LMO. To evaluate the ethanol contents of samples by GC, a mix standard solution with $320 \mathrm{mg} / \mathrm{dL}$ of methanol and ethanol was prepared from with Merck Company (Darmstadt, Germany) trademark. Also, a newly designed kit produced by Arya Mabna Tashkhis Co., Tehran, Iran, was used to qualitatively detect ethanol content of the samples. This kit is designed based on traditional sulfochromic acid method and contains two reactants (a diluent buffer and one color reactant) and an instruction brochure in the pack.

\section{Procedure of the Kit}

According to the brochure of the kit, $50 \mu \mathrm{L}$ of each sample was poured into a test tube with $1 \mathrm{ml}$ of A reactant (diluent buffer). Then, $1 \mathrm{~mL}$ of $\mathrm{B}$ reactant (color reactant) was added to the test tube and shaken hardly to mix. After one minute, the final result was concluded as positive (turquoise color) or negative (different colors except turquoise or colorless) according to the appeared color.

\section{Study measures}

Key indexes in this study were sensitivity, specificity, Positive Predictive Value (PPV), and Negative Predictive Value (NPV). The sensitivity and specificity of a qualitative test refer to its ability to correctly identify the positive and negative results, respectively. In the diagnostic tests, the PPV and NPV should be proportional with the true positive and negative results, respectively. In this research, the accuracy of the newly designed test measured by two area under the ROC curve. The value of this index can be variable between 0 and 1. An area of 1 represents a perfect test; by decreasing the value of this index the test becomes more worthless. Although, positive and negative likelihood, roc area and chance ratio were calculated in the same real and virtual incidences too, the sensitivity, specificity, PPV and NPV are more discussed in continue.

\section{Results}

Out of 839 samples, $72(8.58 \%)$ cases had not ethanol and the ethanol content of $91(10.85 \%)$ cases was determined less than $30000 \mathrm{mg} / \mathrm{L}(3 \% \mathrm{v} / \mathrm{v})$. In other words, $163(19.43 \%)$ cases were determined as negative outcome (nonalcoholic drink). The most of these samples (676 cases or $80.57 \%$ ) had ethanol more than $3 \%$ $\mathrm{v} / \mathrm{v}$ that was evaluated as positive result (alcoholic drinks). Table 1 shows the analytical quality assurance of the kit including four main parameters (sensitivity, specificity, PPV and NPV).

As it is visible, the kit has maximum $(100 \%)$ or high values in sensitivity, specificity, PPV and NPV. The other analytical quality assurance parameters were seemed suitable as well. The gained results confirmed that the used chemical method had enough validity and could be applied for similar conditions.

\section{Discussion}

Consuming alcoholic beverages is banned in $\operatorname{Iran}(2,19-21)$. Based on Iran laws, the existence of ethanol more than 3\% v/v in a confiscated liquid by police has heavy penalties and its owner must be prosecuted. For this reason, abundant suspected fluids are daily examined in LMO of Iran labs to report their ethanol contents to the judiciary system which is associated with spending a lot of time and money of arrested people. Sometimes or at certain times of the year, all these problems are increased which can be accompanied by methanol intoxication outbreaks too. So, having access to a cheap, rapid, and easy method for screening suspected liquids based on their ethanol contents can be very helpful to avoid wasting time and money.

In this study, the efficacy of a newly designed kit was compared with the GC method (gold standard) by using 839 samples prepared from LMO (Guilan province, Iran) alcohol lab. Due to the novelty of the used kit and lack of similar studies, the comparison of our results with previously published ones is practically impossible and from this point of view, the proposed kit and the related presented data seem to be justly unique.

Generally, the kit has calibrated in such a way that the contents of ethanol more than $3 \% \mathrm{v} / \mathrm{v}$ to be evaluated as positive which will be visible as different intensities of turquoise color. Whereas, other concentrations be considered negative that can be seen as different colors (except turquoise) or colorless. In other words, the value $30000 \mathrm{mg} / \mathrm{L}$ is the cut-off point of the kit.

The examined samples were very different and consisted of a mix of industrial (which were illegally imported to the country), homemade kinds (produced by local winemakers), medical alcohol and etc. Therefore, large amounts of methanol were expected in some of them. GC results showed, three cases $(0.36 \%)$ of them contained huge 
amounts $(700,000 ; 870,000$ and 920,000 mg/L) of methanol (more than European standards) which is very dangerous and was lead to gain false-positive results due to the lack of complete specificity of the sulfochromic method for ethanol. Methanol is cheap and available alcohol that is sometimes sold with along ethanol or alone (instead of it) for more profit and can be caused intoxication $[1,19]$. The mean ethanol content of total 839 samples was $11.29 \mathrm{mg} / \mathrm{L}(0-96.5 \% \mathrm{v} / \mathrm{v})$. This amount in the group of samples with positive results (ethanol concentration more than $3 \% \mathrm{v} / \mathrm{v}$ with $3.1-96.5 \% \mathrm{v} / \mathrm{v}$ of range) and negative outcomes (equal or less than of $3 \% \mathrm{v} / \mathrm{v}$ ), were 21.68 and 0.91 $\mathrm{mg} / \mathrm{L}$, respectively. Generally, the used samples and consequently, gained results can be divided into three groups based on their ethanol contents. The groups 1 and 2 were determined as negative, whereas, the samples of group 3 evaluated as positive.

Accordingly, out of 839 tested samples, 72 (8.58\%) cases had not ethanol absolutely and were appraised negatively (group 1). While 91 $(10.85 \%)$ cases had ethanol equal or less than $30000 \mathrm{mg} / \mathrm{L}(3 \% \mathrm{v} / \mathrm{v})$ that they were evaluated negatively too (group 2). Therefore, 163 (19.43\%) cases were totally diagnosed as negative (nonalcoholic drink). But, $676(80.57 \%)$ cases had ethanol more than $3 \% \mathrm{v} / \mathrm{v}$ that was evaluated as positive result (group 3). In other words, although 767 (91.42\%) cases contained ethanol, 676 (80.57\%) were evaluated as alcohol with $21.68 \mathrm{mg} / \mathrm{L}$ mean of ethanol concentration (alcoholic drinks), while the mean of it in other countries is more than it (35-40\% v/v) [22] However, some of the examined samples had high contents of ethanol (above $70 \%$ vol.) that can be due to their medical origin.

As is demonstrated in Table 1, the sensitivity and NPV of used kit were $100 \%$ that is very ideal for a screening kit. The high sensitivity (100\%) indicates the negative obtained results are fully acceptable and so, has definite diagnostic value which is in complete compliance with the NPV result (100\%). These results represent the high ability of the kit in detection of negative samples. Therefore, obtaining false negative result by this kit is impossible. On the other hand, the specificity and PPV of kit are high (Table 1) which are acceptable for a screening kit, however, it is deductible; gaining false positive result by this kit is possible. Generally, according the screening nature of the examined kit, the attained results are very ideal because, in use of it, all samples with negative results will be removed from daily investigation process and the cases with positive results will be re-examined with confirming methods (like GC) to reach the final conclusion.

In addition, it should be noted that although this method is nonspecific and reacts with some other organic compounds (such as Table 1: Validation of the kit.

\begin{tabular}{|c|c|c|}
\hline \multirow{2}{*}{} & $\begin{array}{c}\text { With real incidence } \\
\mathbf{( 6 0 \% )}\end{array}$ & $\begin{array}{c}\text { With virtual incidence } \\
\mathbf{( 1 0 \% )}\end{array}$ \\
\cline { 2 - 3 } & Percent \& 95\% Cl & Percent \& 95\% Cl \\
\hline Sensitivity & $100(99.4 ; \mathbf{1 0 0 )}$ & $100(99.4 ; 100)$ \\
\hline Specificity & $74.1(67.2 ; 80.5)$ & $74.1(67.2 ; 80.5)$ \\
\hline PPV & $93.5(91.4 ; 95.2)$ & $30.2(25.2 ; 35.7)$ \\
\hline NPV & $100(97.3 ; 100)$ & $100(97.3 ; 100)$ \\
\hline Roc Area & $0.872(0.839 ; 0.904)$ & $0.872(0.839 ; 0.904)$ \\
\hline Likelihood Ratio $(+)$ & $3.89(3.03 ; 4.99)$ & $3.89(3.03 ; 4.99)$ \\
\hline Likelihood Ratio $(-)$ & - & - \\
\hline Chance Ratio & - & \\
\hline
\end{tabular}

acetone, acetaldehyde and methanol), repetition of positive tests by confirmatory methods (like GC) prevent their possible effects. The used kit falsely detected 28 (3.3\%) cases with ethanol content more than $3 \% \mathrm{v} / \mathrm{v}$, which was not confirmed by GC (False positive). These inaccurate positive results may be due to existence of mentioned interfering organic compounds in the examined samples. Overall, to the best of our knowledge, in spite of many valuable reports on different aspects of ethanol, no study has been presented about easy and cheap methods for ethanol screening in liquids and the suggested kit has enough sensitivity for separating samples with ethanol more than $3 \% \mathrm{v} / \mathrm{v}$ from the samples with ethanol equal $3 \% \mathrm{v} / \mathrm{v}$ or less than it.

\section{Conclusion}

In Iran black market of alcoholic beverages, the ethanol degree is lower than other countries and maybe contains methanol concentrations higher than the maximum recommended by EU. Also, our results suggest that the newly designed kit is sufficiently rapid and easy and has enough sensitivity and efficacy for screening of ethanol content of beverages. Negative results of this kit indicate lack of ethanol or existence of it up to $3 \% \mathrm{v} / \mathrm{v}$ and have definite diagnostic value, albeit confirmation test (like GC) is needed for validation of the gained positive results.

\section{Acknowledgement}

The authors are grateful to the Legal Medicine Research Center of Iran and Legal Medicine Organization (LMO) of Guilan province for their financial and official supports.

\section{References}

1. Zamani N, Rafizadeh A, Hassanian-Moghaddam $\mathrm{H}$, Akhavan-Tavakoli A Ghorbani-Samin M, Akhgari M, et al. Evaluation of methanol content of illegal beverages using GC and an easier modified Chromotropic acid method; a cross sectional study. Substance Abuse Treatment, Prevention, and Policy. 2019; 14: 56-63.

2. Ghadirzadeh MR, Rafizadeh A, Fatahi A, Mirtorabi SD, Nazari H, Rafizadeh M. Introducing a New Kit based on Modified Chromotropic Acid Method for Easy Determination of Methanol. American Journal of Pharmacology and Toxicolog. 2019; 14: 1-6.

3. Hassanian-Moghaddam H, Zamani N. A Brief Review on Toxic Alcohols: Management Strategies. Iran J Kidney Dis. 2016; 10: 344-350.

4. Hassanian-Moghaddam $H$, Mirafzal A, Nikfarjam A, Saberinia A, Nasehi AA Masoumi-Asl $\mathrm{H}$, et al. Methanol Mass Poisoning in Iran: Role of Case Finding in Outbreak Management. Journal of public health. 2014; 37: 354-359.

5. Rafizadeh A, Shariati Sh, Safarzadeh Vishekaei MN. Determination of herbal distillates methanol with a special diagnostic kit. Journal of Guilan University of Medical Sciences. 2016; 24: 61-67.

6. Saadat F, Hassanian Moghadam H, Zamani N, Rafizadeh A. Quantification of Some Herbal Distillates' Methanol to Evaluate a New Diagnostic Kit. Journal of Food Quality. 2020; 2020.

7. Savary BJ, Nũnez A. Gas chromatography-mass spectrometry method for determining the methanol and acetic acid contents of pectin using headspace solid-phase micro-extraction and stable isotope dilution. Journal of Chromatography A. 2003; 1017: 151-159.

8. Rafizadeh A, NasiriFard R, Nasoori Gazani M, Haghshanas M, Jmali Bivarzani F, Poormohammad L. The effectiveness of whole concentration of homemade herbal distillates on the result of qualitative methanol detection by the chromotropic acid method. Journal of Ornamental and Horticultural Plants. 2013; 3: 105-109.

9. Nasouri Gazani M, Shariati Sh, Rafizadeh A. Using an Easy and Fast 
Modified Chemical Method for Detection of Methanol Content in Drinking Herbal Distillates. Journal of Analytical \& Pharmaceutical Research. 2017; 6: 1-6.

10. Saadat F, Rafizadeh A. Rapid determination of methanol in herbaceous distillates for their safety evaluation by a new modified chromotropic acid method. Iranian Journal of Pharmaceutical Research. 2019; 18: 696-703.

11. Wang ML, Wang JT, Choong YMJ. A rapid and accurate method for determination of methanol in alcoholic beverage by direct injection capillary gas chromatography. J Food Compost Anal. 2004; 17: 187-196.

12. Li Q, H Zhang. Study of methanol catalyzed reaction between sodium 1 , 2-naphthoquine-4-sulfonate and hydroxyl ion and its application in the determination of methanol. Spectrochimica Acta Part A. Molecular and Biomolecular Spectroscopy. 2008; 71: 245-251.

13. Bhosale Y, Mulani Kh, Rathod V. A Rapid and Precise Titration Method for Analysis of Ethanol: Comparison among Serum-Whole Blood Alcohol Concentration. Open Acc Blood Res Trans J. 2017; 1: 1-3.

14. Mason KP, Michna E, Zurakowski D, Koka BV, Burrows PE. Serum ethano levels in children and adults after ethanol embolization or sclerotherapy for vascular anomalies. Radiology. 2000; 217: 127-132.

15. Degel F, Paulus DN. A rapid and precise method for the quantitation of ethanol in serum using the EMIT StTM serum ethanol assay reagent and a cobas bio centrifugal analyzer. Clin Chem Clin Biochem. 1988; 26: 351-353.
16. Wigmore JC, Elliot M. Serum, blood and breath alcohol results in a case of impaired driving causing bodily harm. Canadian Society of Forensic Science Journal. 2004; 37: 223-227.

17. Penetar DM, McNeil JF, Ryan ET, Lukas SE. Comparison among plasma, serum, and whole blood ethanol concentrations: Impact of storage conditions and collection tubes. J Anal Toxicol. 2008; 32: 505-510.

18. Winek CL, Wahba WW, Windisch RM, Winek JrCL. Serum alcohol concentration in trauma patients determined by immunoassay versus gas chromatography. Forensic Sci Int. 2004; 139: 1-3.

19. Hassanian-Moghaddam H, Rafizadeh A, Shariati Sh, Rafizadeh M, Zamani $\mathrm{N}$. Evaluation of methanol content of beverages using an easy modified chromotropic acid method. Food and Chemical Toxicology. 2018; 121: 11-16

20. Ghoreishi SM, Shahbazi F, Mirtorabi SD, Ghadirzadeh MR, Hashemi Nazari SS. Epidemiological study of mortality rate from alcohol and illicit drug abuse in Iran. Journal of research in health sciences. 2017; 14.

21. Shahbazi F, Mirtorabi SD, Ghadirzadeh MR, Hashemi-Nazari SS. Analysis of mortality rate of illicit substance abuse and its trend in five years in Iran, 2014-2018. Addiction \& health. 2018; 4: 260-268.

22. Lachenmeier DW, Sarsh B, Rehm J. The composition of alcohol products from markets in Lithuania and Hungary, and potential health consequences: a pilot study. Alcohol and Alcoholism. 2008; 44: 93-102. 